Fetal Diagnosis and Therapy

\title{
Monochorionic Triplets Complicated by Fetofetal Transfusion Syndrome: A Case Series and Review of the Literature
}

\author{
Suzanne H.P. Peeters ${ }^{a} \quad$ Johanna M. Middeldorp ${ }^{a}$ Enrico Lopriore ${ }^{b}$ \\ Frans J. Klumper ${ }^{\text {a }}$ Dick Oepkes ${ }^{\text {a }}$ \\ Departments of a Obstetrics and ${ }^{b}$ Pediatrics, Leiden University Medical Center, Leiden, The Netherlands
}

\section{Key Words}

Monochorionic/dichorionic triplets • Fetofetal transfusion • Laser therapy · Twin-to-twin transfusion syndrome

\begin{abstract}
Objective: To compare perinatal outcome in monochorionic (MC) triplets with twin-to-twin transfusion syndrome (TTTS) versus dichorionic (DC) triplets with TTTS. Study Design: Retrospective analysis of all triplets with TTTS treated at our center and all cases reported in the literature between 1990 and 2010. Survival and gestational age at birth of MC and DC triplets were compared and stratified by type of intervention. We excluded triplets with one or more fetal deaths $<16$ weeks' gestation and those with one or more fetuses with congenital anomalies. Results: MC triplets were affected by TTTS in a total of 27 cases, and overall survival was 51\% (38/75 fetuses) compared to 105 DC triplets with a survival of $76 \%(220 / 291$ fetuses) $(p<0.05)$. Mean gestational age at birth in MC triplets was 28 weeks, compared to 31 weeks in DC triplets $(p<0.05)$. Perinatal survival of at least one fetus in MC triplet and DC triplet pregnancies was 70\% (19/27) and $91 \%(96 / 105)(p<0.05)$. In DC triplets, survival after laser therapy was significantly improved compared to expectant management, amniodrainage or selective feticide $(p<0.05)$.
\end{abstract}

Conclusion: MC triplets with TTTS are at a considerably higher risk for perinatal mortality and preterm birth than DC triplets. The optimal strategy to manage $M C$ triplets with TTTS, including the role of selective feticide and laser therapy of all anastomoses, is still to be established.

Copyright $\odot 2012$ S. Karger AG, Basel

\section{Introduction}

Twin-to-twin transfusion syndrome (TTTS) is a serious complication of monochorionic (MC) twin gestations and is the result of an unbalanced exchange of blood due to vascular anastomoses that connect the circulations. If untreated, this condition is associated with high perinatal mortality and morbidity rates. Survival is improved with fetoscopic laser coagulation of the placental anastomoses [1]. TTTS is not only seen in MC twins, but can also occur in MC and dichorionic (DC) triplet gestations.

Spontaneous triplets occur in about 1 in 7,000 deliveries, but incidences have increased over the past decades,

Part of this study was presented as a poster at the SMFM 2011, San Francisco, Calif., USA.

\section{KARGER}

Fax +4161306 1234

E-Mail karger@karger.ch

www.karger.com (c) 2012 S. Karger AG, Basel

$1015-3837 / 12 / 0324-0239 \$ 38.00 / 0$

Accessible online at:

www.karger.com/fdt
Suzanne Peeters

Department of Obstetrics, Leiden University Medical Center

K6-35, PO Box 9600

NL-2300 RC Leiden (The Netherlands)

Tel. +31 71526 2896, E-Mail s.h.p.peeters@lumc.nl 
mainly because of assisted reproductive technologies [2]. Depending on the type of placentation, triplet pregnancies can be trichorionic triplets (i.e. 3 fetuses with separate placentas and amniotic cavities), DC triplets (i.e. monochorionic twins and a singleton with a separate placenta) and MC triplets (i.e. 3 fetuses with one shared placenta and three amniotic cavities). Only a few cases of MC (triamniotic) triplets have been reported, with estimated incidences of 1-1.6 in 100,000 deliveries [3, 4]. Since trichorionic triplets have no placental vascular anastomoses, TTTS may only occur in MC and DC triplets. The incidence of TTTS in MC twins is approximately 9\% of all cases [5], and this is probably similar for DC triplets. However, the incidence of TTTS in MC triplets, in which all 3 fetuses shared a common circulation, is not well known.

Management options and survival of triplets with TTTS are frequently discussed in the literature [2, 6-9]. Because an MC twin pair can also be part of any other high-order multiple pregnancy, survival rates of MC triplets are often confused with DC triplets, with one pair of MC twins affected by TTTS and one singleton. The prognosis of TTTS for MC triplets is considered to be different from that for DC triplets and has been reported to be severe, with higher rates of mortality and preterm birth, despite interventions $[7,10]$. However, the literature on triplets and higher-order multiple births with TTTS consists mainly of case reports and small series, most of which fail to discuss placentation [3, 11-19].

In this study, we aim to compare the perinatal outcomes of all the MC and DC triplets with TTTS treated at our center and reported in the literature in the last two decades.

\section{Material and Methods}

At the Leiden University Medical Center (LUMC), the national tertiary referral center for invasive fetal therapy, fetoscopic laser surgery has been the preferred treatment modality for all pregnancies complicated by TTTS Quintero stage II or higher, and for selected cases with Quintero stage I with symptomatic polyhydramnios since August 2000 [20].

\section{Cases in LUMC}

We performed a retrospective analysis of prospectively collected data on perinatal outcome and management of all MC and DC triplet pregnancies with TTTS treated at our center. An MC triplet pregnancy was diagnosed when the 3 fetuses shared the same MC placenta, and a DC triplet pregnancy was determined when 2 fetuses shared one MC placenta, whereas the third fetus had an independent single placenta.

Detailed sonograpic examination was performed in all fetuses at the referral center and continued on a weekly or biweekly basis at our center. Chorionicity and amnioniticy were established by sonographic examination in the first trimester of pregnancy. The diagnostic (established) criteria for TTTS in triplets were similar to that for twins. Details on sonographic criteria and details on procedures for fetoscopic laser surgery and umbilical-cord coagulation have been described previously [20]. Once the diagnosis of TTTS was established, parents were counseled regarding the outcome and offered the options of expectant treatment, termination of the whole pregnancy, serial amniodrainage, selective termination of (one of) the MC twins in cases of DC triplets, or 1 of the fetuses in the case of lethal prognosis or fetoscopic coagulation of the communicating vascular anastomoses. After written informed consent was obtained from the parents, the procedure was performed by one of the four operators, specialized in fetoscopic surgery, as previously reported [20], using a fetoscope (2 mm; Storz, Vianen, the Netherlands) for percutanous introduction through a 3.3-millimeter shaft into the amniotic sac of the recipient(s). Coagulation of the anastomoses or umbilical cord was performed using a diode laser (Diomed Limited, Cambridge, $\mathrm{UK}$ ) or Nd:YAG laser (Dornier Medizin Technik, Germering, Germany) with an output of 15-70 W. If there were two recipients in MC triplets, the fetoscope was introduced into both cavities via two different uterine entries, but if there were two donors, only the vascular anastomoses between the recipient and both donor fetuses were coagulated.

After the procedure, patients remained in the hospital for 12 $48 \mathrm{~h}$. Complications including vaginal blood loss, hypotension, bleeding from uterine vessels, prelabor premature rupture of membranes (PPROM) and maternal fever were recorded. Ultrasound examination was performed within $24 \mathrm{~h}$ after surgery and then on a weekly or biweekly basis. The data on obstetric and neonatal outcomes were derived from medical charts. In cases where the delivery did not take place in our center, data were provided by outcome reports from the referring obstetricians and pediatricians.

\section{Meta-Analysis of Cases Reported in the Literature}

An electronic literature search using MEDLINE, EMBASE and the Cochrane Database was performed to find all relevant articles reporting perinatal outcome and management in $\mathrm{MC}$ and DC triplet pregnancies with TTTS. A search in PubMed was performed to find relevant articles reporting outcomes of MC and DC triplet pregnancies complicated by TTTS using the following keywords 'fetofetal transfusion' AND 'triplet', 'survival rate', 'monochorionic' AND 'laser therapy' OR 'intervention'. No language restrictions were applied. We accepted original articles, short communications, letters to the editor and case reports. In addition, a search was performed from the reference list of all identified articles. When needed, we contacted authors for additional, unpublished information. Articles were included irrespective of their primary objective. We included all reported cases of MC and DC triplet pregnancies assessed by 1st-trimester ultrasound and complicated by TTTS. TTTS was diagnosed with standard criteria (polyhydramnios $>8 \mathrm{~cm}$ in the recipient(s) $\mathrm{sac}(\mathrm{s})$ and oligohydramnios $<2 \mathrm{~cm}$ in the donor sac). Exclusion criteria were one or more fetal deaths $<16$ weeks' gestation and pregnancies with 1 or more fetuses with congenital anomalies. Cases in which artificial termination of the whole pregnancy was performed were not included.

Two of the authors (S.H.P.P. and D.O.) initially screened all the titles and abstracts of papers, identified by the review search strat- 
egy, for their relevance. Only studies that were obviously irrelevant were excluded at this stage; they independently assessed all other studies independently (on the basis of their full text) for inclusion or exclusion, using the above criteria. Discrepancies were to be resolved by discussion with a third reviewer, but this proved unnecessary.

Data extracted from each article included survival, gestational age at birth, intervention, and, if reported, long-term perinatal outcomes in donors and recipients. A p value $\leq 0.05$ was considered to indicate statistical significance. A $\chi^{2}$ test and an independent Student $t$ test were used to compare survival and gestational age between triplets. Statistical analysis was performed with SPSS version 18.0 (SPSS, Inc., Chicago, Ill., USA).

\section{Results}

\section{Cases in LUMC}

During the 10-year study period from 2000 to 2010,10 fetoscopic procedures in $2 \mathrm{MC}$ and $8 \mathrm{DC}$ triplet pregnancies were performed in our center. All triplet pregnancies were triamniotic.

A total number of 340 laser surgeries were performed for all twin and triplet pregnancies affected by TTTS during this 10 -year period. Details on gestational age at procedure, type of intervention and outcome in triplet pregnancies are described in table 1.

Fetoscopic laser coagulation was performed in 9 cases, including 1 case of MC triplets with percutaneous entry into two amniotic sacs. In 1 case of MC triplets, umbilical cord coagulation with selective reduction of the recipient was carried out. The procedure was performed at a median gestational age of 18 weeks (range 14-26 weeks). Three cases were classified as Quintero stage II and 7 cases Quintero stage III. Fetal demise occurred in 9 of 30 fetuses (30\%) (including 2 cases of selective feticide) and neonatal demise in 1 neonate (3\%). Overall perinatal survival was $70 \%(20 / 30)$. In the DC triplets, perinatal survival of both children involved in the monochorionic twin pair was 50\% (4/8). Median gestational age at delivery was 31 weeks' gestation (range 21-36 weeks). Median birth weight in live-born neonates was 1,661 g (range $845-2,780 \mathrm{~g})$.

A procedure-related complication occurred in 1 case (table 1, case No. 3) where laser coagulation of a DC triplet was performed. During uterine entry, an umbilical artery of the donor was perforated and massive bleeding occurred, with the result of intrauterine fetal demise of the donor. The recipient twin and the singleton were born at 34 weeks' gestation. No maternal complications occurred.

In one of the DC triplets (table 1, case No. 7) treated with laser surgery at 20 weeks' gestation, intrauterine fe- tal demise of the donor was assessed at 22 weeks. PPROM occurred at 27 weeks' gestation and corticosteroids, antibiotics and atosiban were administered to prevent preterm labor. Nevertheless, the patient went into spontaneous labor at 29 weeks and 6 days. The MC twin in this pregnancy was delivered at 30 weeks' gestation, including the stillborn donor, but the recipient died 4 days after birth due to intracranial hemorrhage grade IIb and sepsis due to group B streptococcus. The delivery of the third child took place on the same day and it was alive and well at discharge.

In 1 case of DC triplets (table 1, case No. 9) fetoscopic laser coagulation was performed with intentional perforation of the intertwin membrane because of the presence of vascular anastomoses in the other amniotic cavity, thereby creating a monoamniotic twin and one singleton. Unfortunately MRI scans that were performed during follow-up of the pregnancy showed severe brain damage with ventriculomegaly and ischemic lesions of the former recipient. A reintervention was performed and selective feticide of the recipient twin was carried out. The donor and the singleton were born healthy at 35 weeks' gestational age.

\section{Meta-Analysis of Cases Reported in the Literature}

From 1990 to 2010, 22 articles met the inclusion criteria and we identified $25 \mathrm{MC}$ and $97 \mathrm{DC}$ triplet pregnancies with TTTS [2, 3, 6-19, 21-25]. Quintero stage, in the reported cases including the cases in this study, was stage I in $7 \%(2 / 27)$ stage II in $15 \%(4 / 27)$, stage III in $45 \%$ $(12 / 27)$ and stage IV in 33\% (9/27) of the MC triplets. In the DC triplets, Quintero stage I was classified in $2 \%$ $(2 / 105)$, stage II in $23 \%(24 / 105)$, stage III in $68 \%(72 / 105)$ and stage IV in $7 \%(7 / 105)$.

Survival rates of all triplet pregnancies complicated by TTTS reported in the literature (including the cases in our center) - classified according to chorionicity - are presented in table 2. Perinatal survival of at least 1 fetus in MC and DC triplet pregnancies was 70\% (19/27) and $91 \%(96 / 105)$, respectively $(\mathrm{p}=0.004)$. Overall perinatal survival of MC and DC triplets was 51\% (38/75) and 76\% $(220 / 291)$, respectively $(p=0.018)$. Median gestational age at delivery was 28 weeks (range 18-40 weeks) for the MC triplets, compared to 31 weeks (range 20-39 weeks) in the DC triplets $(\mathrm{p}=0.016)$. In DC triplets, survival after laser therapy was significantly improved compared to expectant management, amniodrainage or selective feticide $(\mathrm{p}=0.007)$. Neonatal death within 4 weeks after birth occurred in $8 \%(6 / 75)$ of $\mathrm{MC}$ cases and in $8 \%$ $(24 / 291)$ of DC cases $(p=N S)$. In 1 case of MC triplets, a neonate died on the 75th day after birth. 


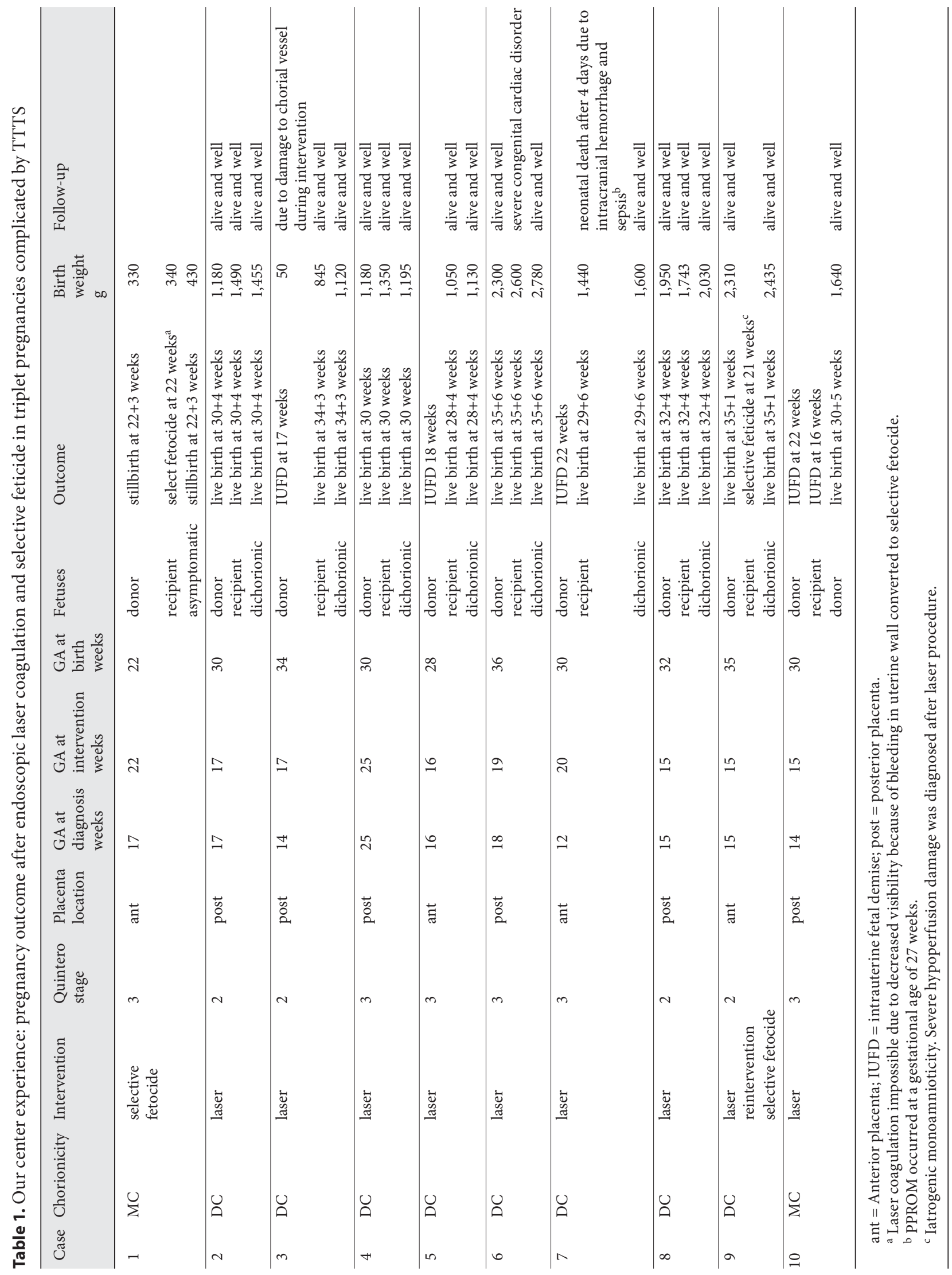




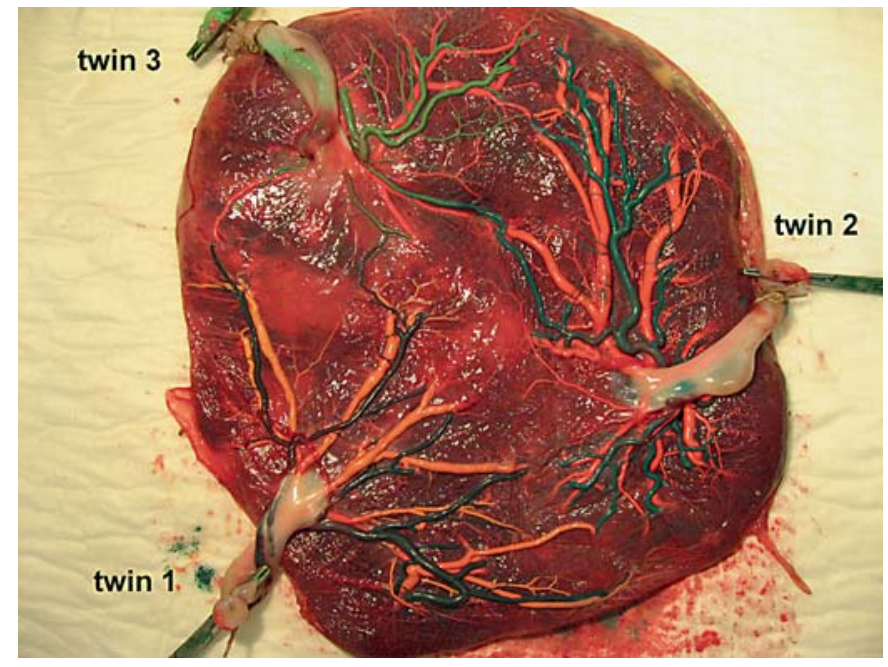

Fig. 1. Placenta of MC triplets with vascular anastomoses.

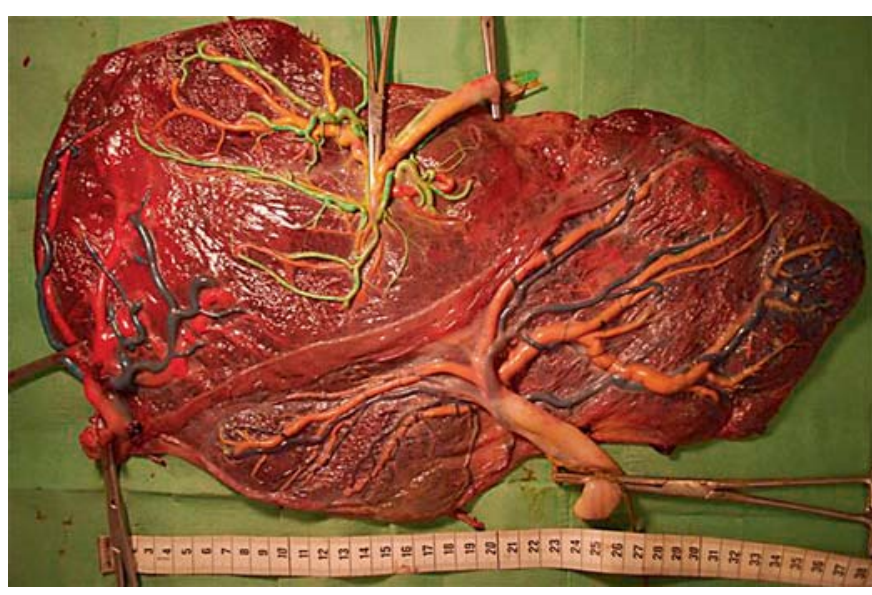

Fig. 2. Placenta of DC triplet with MC twin complicated by TTTS and treated with laser surgery and one singleton. As is common in dichorionic placentas, the placenta of the singleton is fused together with the monochorionic twin placenta. The thick intertwin membrane indicates dichorionicity.

Table 2. Survival rates and gestational age at birth of MC and DC triplet pregnancies according to type of intervention

\begin{tabular}{|c|c|c|c|c|c|c|}
\hline \multirow[t]{2}{*}{ Intervention } & \multicolumn{4}{|c|}{ Survival (fetuses) } & \multicolumn{2}{|c|}{ Gestational age at birth weeks (range) } \\
\hline & MC triplets & $\mathrm{n}$ & DC triplets & $\mathrm{n}$ & MC triplets & DC triplets \\
\hline Laser & $26 / 48(54 \%)$ & 16 & $184 / 237(78 \%)$ & 79 & $28(18-34)$ & $31(20-36)$ \\
\hline Selective feticide & $1 / 6(17 \%)$ & 2 & $11 / 24(46 \%)$ & 8 & $31(22-40)$ & $32(25-37)$ \\
\hline Amniodrainage & $4 / 9(44 \%)$ & 3 & $20 / 39(51 \%)$ & 13 & $26(23-27)$ & $29(24-39)$ \\
\hline Expectant management & $7 / 18(39 \%)$ & 6 & $5 / 15(33 \%)$ & 5 & $28(23-35)$ & $26(23-29)$ \\
\hline Total & $38 / 81(47 \%)$ & 27 & $220 / 315(70 \%)$ & 105 & $28(18-40)$ & $31(20-39)$ \\
\hline
\end{tabular}

Figures 1 and 2 demonstrate the difference between a triplet pregnancy with an MC placenta with vascular anastomoses and a DC placenta of an MC twin (and one singleton) complicated by TTTS and treated with laser coagulation.

\section{Discussion}

This study shows that perinatal morbidity and mortality in MC triplets with TTTS is higher than in DC triplets. Of the 81 fetuses involved in fetofetofetal transfusion, only 38 (51\%) survived. In addition, median gestational age at delivery in $\mathrm{MC}$ triplet pregnancies is significantly shorter thanin DC triplets (28 vs. 31 weeks' gestation). This outcome could be due to the technical difficulties of interventions in $\mathrm{MC}$ triplets because all three circulations are connected, therefore making fetoscopic treatment much more challenging.

The data in this study also demonstrate that fetoscopic laser coagulation of communicating vascular anastomoses in DC triplet pregnancies complicated by TTTS is a feasible treatment option with increasing survival rates and more advanced gestational age at birth. Perinatal survival rates of at least one survivor $(91 \%)$ appear comparable to MC twins (around 90\%) [26, 27]. Although treatment appears to be associated with an improved perinatal outcome for DC triplets, survival rates are still limited for MC triplets. 
Various therapeutic measures have been proposed for triplets with TTTS. Amnioreduction, to decrease the pressure of the amniotic cavity with polyhydramnios, is not a permanent solution and is associated with a high risk of mortality and morbidity to the cotriplets due to the presence of vascular anastomoses in the case of intrauterine fetal demise. Serial amnioreduction is therefore considered, at best, a temporary treatment and does not correct the underlying problem.

Fetoscopic laser coagulation is the only causative treatment option because, provided that all anastomoses can be visualized on the chorionic surface, this operation will separate the fetal circulations [28]. This technique potentially avoids perinatal mortality and the high morbidity rate attributed to vascular accidents mainly after intrauterine death of one of the multiplets [7]. Loss of cotyledons following laser ablation could either cause or exacerbate placental insufficiency in the normal triplets; however, according to Chmait et al. [29], twin weight discordance and donor fetus intrauterine growth restriction appear to improve after laser therapy. In DC triplet pregnancies with severe TTTS, the technique for fetoscopic laser coagulation is the same as in MC twins with this condition. Comparable to Sepulveda et al. [7], survival rates appear similar to that of MC twins when a successful procedure was performed. Conversely, in $\mathrm{MC}$ triplet pregnancies the outcome was poor. In 6 cases of MC $[3,6,9,11,15,22]$ and 5 cases of DC $[6,23,24]$ triplets complicated by TTTS, only $12 / 33$ (36\%) survived with expectant management. Although based on small numbers, these results strongly suggest that laser coagulation is preferable to expectant management or amniodrainage [17]. Five successful cases of MC triplet gestations (with 3 survivors) treated with laser coagulation were reported (including the cases in our study) $[2,7-10,21]$, but 11 other cases were not successful.

Most likely, this is due to the technical difficulties of the fetoscopic treatment because of the identification and coagulation of vascular anastomoses between all 3 fetuses. To reach the appropriate location of the interfetal communicating vessels, it is necessary to have two uterine entries with the fetoscope into two different sacs. In addition, complete ablation of the intercommunications can be technically impossible to achieve because of placental location, the presence of multiple fetal parts that hamper visualization of the placental anastomoses and oligohydramnios in the sac of the donor. Incomplete separation could result in the intrauterine death or severe morbidity of the remaining fetus(es) after the procedure, because of retrograde hemorrhage of the live fetuses into the dead placental tissue.
Selective feticide of the affected triplets [i.e. the MC pair in a DC triplet or a compromised triplet(s)] offers the potential advantages of interrupting the circulatory imbalance with a single procedure and provides a maximal placental volume for the remaining fetus. Umbilical cord coagulation has proven beneficial in conditions such as twin-reversed arterial perfusion or MC twins discordant for a major anomaly, with survival of $78 \%$ of co-twins and an excellent long-term outcome; therefore, it may also be used in triplets with these kinds of conditions [30]. Furthermore, some parents could consider selective reduction of 1 or more healthy fetuses, to improve the survival and long-term follow-up of the remaining children.

Care should be taken when interpreting these results due to the limited data on perinatal outcome in triplets with TTTS, particularly MC triplets. Only 27 cases of MC triplet pregnancies with TTTS have been reported in the literature. The actual number of $\mathrm{MC}$ and DC triplets with TTTS may be higher due to underreporting/publication bias. Several cases in which the pregnancy was terminated or fetal demise occurred spontaneously have probably not been reported. Irrespective of zygocity, triplets are high-risk pregnancies due to the high incidence of preterm delivery, intrauterine growth restriction and congenital anomalies [31]. Only 1 case of fetofetal transfusion syndrome in MC quadruplets has ever been reported. Laser ablation of vascular anastomoses between one donor and two recipients was carried out at 20 weeks' gestation and fetal demise of one quadruplet occurred shortly after procedure. Delivery occurred at 25 weeks after the recurrence of TTTS. Only the sole survivor, not affected by transfusion, was born alive [32].

In conclusion, this case series and review of the literature demonstrates that, although technically more challenging, fetoscopic surgery is feasible in triplets. In cases of MC triplets where fetoscopic laser coagulation is technically impossible, parents should be counseled on the increased likelihood of unfavorable outcome. However, the rarity of these conditions, the required operator and prenatal diagnostic skills, the variety of management options and the requirement of in-depth counseling of patients currently limit the availability of such interventions to referral centers for fetal medicine. Further experience will be required in order to evaluate the risks and efficacy of this therapy in triplet cases. 


\section{References}

1 Yamamoto M, Ville Y: Recent findings on laser treatment of twin-to-twin transfusion syndrome. Curr Opin Obstet Gynecol 2006; 18:87-92.

$>2$ Van Schoubroeck SD, Lewi L, Ryan G, Carreras E, Jani J, Higueras T, et al: Fetoscopic surgery in triplet pregnancies: a multicenter case series. Am J Obstet Gynecol 2004;191: 1529-1532.

$>3$ Entezami M, Runkel S, Becker R, Weitzel $\mathrm{HK}$, Arabin B: Feto-feto-fetal triplet transfusion syndrome (FFFTTS). J Matern Fetal Med 1997;6:334-337.

4 Savelli L, Gabrielli S, Pilu G: Two- and threedimensional sonography of a monochorionic triplet gestation. Ultrasound Obstet Gynecol 2001; 18:683-684.

$>5$ Lewi L, Jani J, Blickstein I, Huber A, Gucciardo L, Van MT, et al: The outcome of monochorionic diamniotic twin gestations in the era of invasive fetal therapy: a prospective cohort study. Am J Obstet Gynecol 2008; 199:514-518.

$\checkmark 6$ Hayashi A, Kikuchi A, Joshita N, Matsumoto Y, Tatematsu M, Horikoshi T, et al: Monochorionic triplet pregnancy complicated by severe fetofetal transfusion. J Obstet Gynaecol Res 2005;31:414-420.

$>7$ Sepulveda W, Surerus E, Vandecruys H, Nicolaides KH: Fetofetal transfusion syndrome in triplet pregnancies: outcome after endoscopic laser surgery. Am J Obstet Gynecol 2005;192:161-164.

$>8$ De Lia JE, Worthington D, Carr MH, Graupe $\mathrm{MH}$, Melone PJ: Placental laser surgery for severe previable feto-fetal transfusion syndrome in triplet gestation. Am J Perinatol 2009;26:559-564.

$\checkmark 9$ Diemert A, Diehl W, Huber A, Glosemeyer P, Hecher K: Laser therapy of twin-to-twin transfusion syndrome in triplet pregnancies. Ultrasound Obstet Gynecol 2010;35:71-74.

$\checkmark 10$ Chmait RH, Kontopoulos E, Bornick PW, Maitino T, Quintero RA: Triplets with fetofetal transfusion syndrome treated with laser ablation: the USFetus experience. J Matern Fetal Neonatal Med 2010;23:361-365.

-11 Fisk NM, Borrell A, Hubinont C, Tannirandorn Y, Nicolini U, Rodeck CH: Fetofetal transfusion syndrome: do the neonatal criteria apply in utero? Arch Dis Child 1990;65: 657-661.
12 Rehan VK, Menticoglou SM, Seshia MM, Bowman JM: Fetofetal transfusion in triplets. Arch Dis Child Fetal Neonatal Ed 1995; 73:F41-F43.

13 Sohn C, Wallwiener D, Kurek R, Hahn U, Schiesser M, Bastert G: Treatment of the twin-twin transfusion syndrome: initial experience using laser-induced interstitial thermotherapy. Fetal Diagn Ther 1996;11: 390-397.

14 Ling PY, Leo MV, Rodis JF, Campbell WA: Amnioreduction in triplet fetofetal transfusion. Obstet Gynecol 2000;96:843.

15 Giles W, O’Callaghan S, Cole S, Bisits A: Triplet pregnancy complicated by feto-fetofetal transfusion with very rapid deterioration and fetal demise in all three triplets. Aust N Z J Obstet Gynaecol 2002;42:408409.

16 Taylor MJ, Shalev E, Tanawattanacharoen S, Jolly M, Kumar S, Weiner E, et al: Ultrasound-guided umbilical cord occlusion using bipolar diathermy for Stage III/IV twintwin transfusion syndrome. Prenat Diagn 2002;22:70-76.

17 Leung WC, Wong KY, Leung KY, Lee CP, Tang MH, Lao TT: Successful outcome after serial amnioreductions in triplet fetofetal transfusion syndrome. Obstet Gynecol 2003; 101:1107-1110.

18 Baschat AA, Muench MV, Mighty HE, Harman CR: Successful intrauterine management of severe feto-fetal transfusion in a monochorionic triplet pregnancy using bipolar umbilical cord coagulation. Fetal Diagn Ther 2003;18:397-400.

19 Chasen ST, Al-Kouatly HB, Ballabh P, Skupski DW, Chervenak FA: Outcomes of dichorionic triplet pregnancies. Am J Obstet Gynecol 2002; 186:765-767.

20 Middeldorp JM, Sueters M, Lopriore E, Klumper FJ, Oepkes D, Devlieger R, et al: Fetoscopic laser surgery in 100 pregnancies with severe twin-to-twin transfusion syndrome in the Netherlands. Fetal Diagn Ther 2007;22:190-194.

21 Ishii K, Murakoshi T, Numata M, Kikuchi A, Takakuwa K, Tanaka K: An experience of laser surgery for feto-fetal transfusion syndrome complicated with unexpected fetofetal hemorrhage in a case of monochorionic triamniotic triplets. Fetal Diagn Ther 2006; 21:339-342.

-22 Kruse AJ, Havenith M, Arabin B: Comparison of pregnancy course and outcome with color and radiographic angiography of the placenta in a monochorionic triplet pregnancy. Placenta 2006;27:517-520.
23 Adegbite AL, Ward SB, Bajoria R: Perinatal outcome of spontaneously conceived triplet pregnancies in relation to chorionicity. Am J Obstet Gynecol 2005;193:1463-1471.

24 Geipel A, Berg C, Katalinic A, Plath H, Hansmann $M$, Germer U, et al: Prenatal diagnosis and obstetric outcomes in triplet pregnancies in relation to chorionicity. BJOG 2005; 112:554-558.

25 Young BK, Stephenson CD, Mackenzie AP, Roman AS, Rebarber A, Minior VK, et al: Combined sonographic and endoscopic umbilical cord occlusion in twin and triplet gestations. J Perinat Med 2005;33:530-533.

26 Chmait RH, Kontopoulos EV, Korst LM, Llanes A, Petisco I, Quintero RA: Stagebased outcomes of 682 consecutive cases of twin-twin transfusion syndrome treated with laser surgery: the USFetus experience. Am J Obstet Gynecol 2011;204:393.

27 Baschat A, Chmait RH, Deprest J, Gratacos E, Hecher K, Kontopoulos E, et al: Twin-totwin transfusion syndrome (TTTS). J Perinat Med 2011;39:107-112.

28 Senat MV, Deprest J, Boulvain M, Paupe A, Winer N, Ville Y: Endoscopic laser surgery versus serial amnioreduction for severe twin-to-twin transfusion syndrome. N Engl J Med 2004;351:136-144.

29 Chmait RH, Korst LM, Bornick PW, Allen $\mathrm{MH}$, Quintero RA: Fetal growth after laser therapy for twin-twin transfusion syndrome. Am J Obstet Gynecol 2008;199:47-6.

30 Lewi L, Gratacos E, Ortibus E, Van SD, Carreras E, Higueras T, et al: Pregnancy and infant outcome of 80 consecutive cord coagulations in complicated monochorionic multiple pregnancies. Am J Obstet Gynecol 2006;194:782-789.

31 Papageorghiou AT, Liao AW, Skentou C, Sebire NJ, Nicolaides KH: Trichorionic triplet pregnancies at 10-14 weeks: outcome after embryo reduction compared to expectant management. J Matern Fetal Neonatal Med 2002;11:307-312.

>32 O’Brien BM, Feltovich HM, Carr SR, Luks FI: Feto-fetal transfusion syndrome in monochorionic quadruplets. Obstet Gynecol 2010;115:470-472. 